\title{
Efficient Group Advertising over Public Display Based on User Viewing Time
}

\author{
Yanbin Wang, Yuchun Guo and Yishuai Chen \\ School of Electrical and Information Engineering, Beijing Jiaotong University, Beijing, 100044, China \\ 13120135@bjtu.edu.cn, ycguo@bjtu.edu.cn, yschen@ bjtu.edu.cn
}

Keywords: public display, viewing time, display advertisement, UCB1, group recommendation

\begin{abstract}
Public display appears everywhere providing a variety of services. Advertising is the main revenue source of public display. Existing advertising strategies, however, is inefficient, due to the difficulty of collecting users' explicit feedback, e.g., user rating or click. No implicit feedback method is proposed so far. In this paper, we propose to use users' viewing time to infer audiences' interest in the advertisement. We further design practical algorithms to handle the case that multiple users arrive and watch the public display at the same time. To derive a group's collective interest in an advertisement, we consider the media display structure characteristics of advertisements, as we found users' viewing time of public display is highly related to the advertisement's media display structure. We implemented a system prototype and conducted extensive field experiment to evaluate it. Experimental results demonstrate that users viewing time is a valid implicit feedback to infer audience preference on public display advertisement, and the proposed advertising strategies are practical and effective for group aware advertising.
\end{abstract}

\section{Introduction}

Public displays are replacing static signs to provide audience a variety of services. Advertising is the main revenue source of public displays. For providers of public displays, a key performance metric is the efficiency of advertisement. Existing public display advertisement strategies, however, are inefficient, due to the difficulty of collection of users' feedback on the advertisement and then learn audiences' interest. So far, the vast majority of public display advertisements do not collect user feedback and do personalized optimization.

To achieve more effective and personalized advertising for public display, in this paper, we propose to use users' viewing time of advertisement as implicit feedback of users' interest in the advertisement. Two methods here are introduced to infer each audience's personalized rating based on viewing time, in particular, we propose a novel learning method to infer a user's interest in the playing advertisements during her arriving and watching process. The method has very low computation complexity and fits display advertisement efficiently.

We further design group advertising strategies to handle the case that multiple users arrive and watch the public display at the same time. We study this problem as a group recommendation problem. To derive a group's collective interest in an advertisement, we consider the display structure characteristics of advertisements and introduce a media similarity metric to characterize the media feature similarity between target advertisement and neighbor advertisement. We implemented a system prototype and recruited dozens of persons to conduct extensive experiment. Experimental results show that

- It is accurate to infer a user's interest in a public display advertisement from her viewing time.

- The proposed UCB-like algorithm is efficient to real-time update users' interest on advertisement and has very low computation complexity and thus fit public display efficiently.

- The group advertising strategies can obtain good user advertising experience. In particular, the introduction of media similarity improves the accuracy of advertisement significantly. 


\section{Related Work}

Public display has been widely used in various scenes. Previous research shows that public display indeed not only attracts crowds' attention but achieves a high percent of audience interaction [9]. On the other hand, some other researchers also explored public display and applications and large public display have been combined to encourage people healthy eating in a way of bilateral interaction [4, 11]. However, the above researches are common in requiring audiences to take initiative to interactive with screens. It is not intended for public screens simply offer advertising.

For advertisement on web pages, many previous studies have made contributions. Dwell time on web pages has been proved that it can replace clicks to predict user behavior [14]. But explicit user feedback methods like dwell time are inapplicable for public display advertisement so far. On the other hand, for more than one person, group recommendation is needed. The relationship between members in the group has been taken into consideration to guarantee the maximized correctness of group rating [5]. Furthermore, emotional characteristics are utilized to improve the advertising hits [3]. And image features of static or dynamic images were extracted by image processing technique as content features [6]. Different from these methods, we use structure characteristics to improve our performance, without complex technology like image processing or content extraction.

\section{User Interest Inference}

In order to calculate the rating for an advertisement of a group, firstly we need to know user interest feedback for individual. To exactly record how an audience views an advertisement, we use face detection technology in OpenCV [2] to detect how long an audience watched the playing advertisement. By using multimedia data preprocessing techniques, we get the viewing time set of audiences for different advertisements. Based on the obtained user watching behaviors, we introduce two ways to infer users' interest.

Viewing Time as Personalized Rating. The first method, defined as Viewing Time as Personalized Rating (VT). We use the average viewing time as an audience's viewing time for an advertisement. For example, audience $u$ viewed on advertisement $i$ for once and the viewing time is 4 seconds. Then the viewing time of audience $u$ for advertisement $i$ is defined as $t_{u, i}=4$. On the other hand, if audience $u$ viewed on advertisement $i$ for three times, and the viewing times are 2, 1, 3 seconds. Then $t_{u, i}=2$. After that, we take logarithm of viewing time, and fit the values into 1-5 according to the probability.

Based on the measured user viewing time metric, we get a rating matrix $R$ including rating values of all audiences for all advertisement. As not every audience has viewed every advertisement, we use BiasSVD to fill in the blanks of $R$. More details about BiasSVD, please refer to [13]. After the above process, we get a full rating matrix, defined as $R_{V T}$.

Audience Priority as Personalized Rating. The second method is named as Audience Priority as Personalized Rating (AP), inspired by UCB1 algorithm [1] in multi-armed bandit problem. In this method, we compute advertisements' priority for each audience independently. Specifically, for user $u$, we obtain the priority of advertisement $i$ by

$$
\operatorname{Pr}_{u, i}=\frac{T_{u, i}}{T_{u}}+\sqrt{\frac{2 \log \left(T_{a l l}\right)}{T_{u}}}
$$

where $P r_{u, i}$ denotes the priority value for advertisement $i$ of audience $u$. $T_{\text {all }}$ is a constant meaning a period of time. Here $T_{\text {all }}$ is 852. $T_{u, i}$ denotes the viewing time for advertisement $i$ of audience $u$ in $T_{\text {all }}$, and $T_{u}$ denotes the viewing time for all advertisements of audience $u$ in $T_{\text {all }}$. Note that the calculation of $\mathrm{Pr}_{u, i}$ is based on only audience $u$ 's historical data. As a result, the computation complexity of AP method is considerably lower than that of VT method. For all audiences and all advertisements, a rating matrix can be computed, defined as $R_{A P}$. 


\section{Group Interest Inference}

Based on individual audience's rating, we further compute group rating to deal with the situation when a group of audiences standing in front of the screens. We put audiences in front of the digital screen at the same time as a group, defined as $G$.

For a target advertisement $i_{t}$, we define all the other advertisements as its "neighbor set", named $I_{N}$. Then, we calculate the rating of $i_{t}$ of the group by the group ratings of advertisements in $I_{N}$, we named this method as Neighbor Attractive Rating (NAR). The followings are the detailed steps of NAR.

Step1: Media Similarity Metric. The particularity of the display scene determines that a key point of effective display advertisement is attracting people's attention. Different visual characteristics may strongly affect audiences' attention on advertisement [6]. Therefore, we introduce a media similarity metric to characterize the display similarity between target advertisement and neighbor advertisement. It is given by

$$
D_{i_{t}, i_{n}}=\sum_{f_{i} \in F}\left(\omega_{f_{i}, i_{t}}-\bar{\omega}_{f_{i}}\right)\left(\omega_{f_{i}, i_{n}}-\bar{\omega}_{f_{i}}\right)
$$

where ${ }^{D_{i_{t}, i_{n}}}$ denotes the similarity between target advertisement $i_{t}$ and neighbor advertisement $i_{n}, f$ denotes features in advertisements, $\omega_{f_{i}, i_{t}}$ denotes the percentage of feature $i$ in $i_{t}, \bar{\omega}_{f_{i}}$ denotes the global average percentage of feature $i$. For instance, if 40 percentage of space in $i_{t}$ is occupied by words (f1) and the rest is occupied by pictures (f2), we have $\omega_{f_{1}, i_{t}}=0.4$ and $\omega_{f_{2}, i_{t}}=0.6$.

Step2: Group Rating of "Neighbor Set". We now calculate the group's rating for $i_{n}$ in the "neighbor set" of $i_{t}$. It is the average rating of all members in the group on advertisement. That is:

$$
r_{G, i_{n}}=\frac{\sum_{u \in G} r_{u, i_{n}}}{|G|}
$$

where ${ }_{G, i_{n}}$ denotes the rating for in of group $G,{ }^{r_{u, i_{n}}}$ denotes the rating for $i_{n}$ of audience $u$, and $|G|$ denotes the number of audience in group $G$.

Step3: Group Rating for Target Advertisement. Inspired by Chen et al [9], we calculate the group rating for a target advertisement considering media similarity metric between $i_{t}$ and $i_{n}$, given by

$$
r_{G, i_{t}}=\lambda \bar{r}_{i_{t}}+(1-\lambda) \frac{\sum_{i_{n} \in I_{N}}\left(r_{G, i_{n}}-\bar{r}_{i_{n}}\right) \bullet \mathrm{D}_{i_{t}, i_{n}}}{\left|I_{N}\right|}
$$

where ${ }_{G, i_{t}}$ denotes the rating for it of group G, ${ }^{\bar{r}_{i_{t}}}$ and ${ }^{\bar{r}_{i_{n}}}$ denotes the global average rating for $i_{t}$ and in, $\left|I_{N}\right|$ denotes number of advertisements in $I_{N}$. $\lambda$ is a constant, and here we set it to 0.6.

Through the above three steps, we get group rating for target advertisement.

\section{Group advertising strategies}

Based on above user interest inference method and group interest inference method, we design two group advertising strategies. The strategies can be seen in Fig. 1. Each strategy consists of personalized rating and group rating. For instance, VT-NAR is the combination of VT and NAR, that is, we calculate rating for group using neighbor attractive rating method (NAR), and the personalized rating used in NAR is $R_{V T}$, which is calculated by the Viewing Time as Rating (VT) method. We conducted an experiment of these two strategies. In the next section, we will provide the detailed experiment setup and evaluation results. 


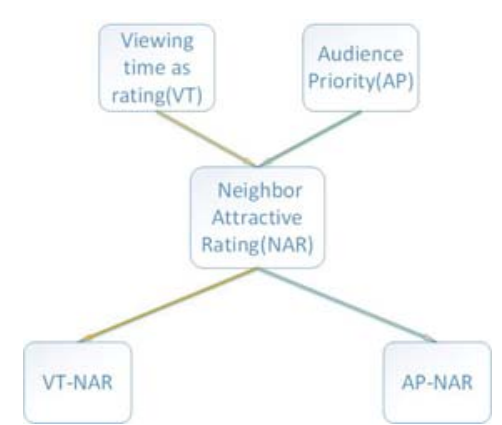

Fig. 1. Proposed group advertising strategies

\section{Experiment Setup and Result}

Dataset. We conducted field experiment in our laboratory. In the end, we obtain an experiment dataset including 42 individuals on the 10 advertisements. 5 seconds is long enough to pull an audience in and show your message [12]. For more accuracy, we set the play time of each advertisement as 10 seconds. The advertisement types include movie, TV series, sports, food, news and shopping. We invited our audiences to rate the advertisements subjectively. For simplicity, we choose words and pictures as media features of advertisement, as shown in Table 1.

Table 1 Media features in advertisements

\begin{tabular}{cll}
\hline \multicolumn{1}{c}{ Percentage } & Words & Pictures \\
\hline Advertisements & & \\
\hline Ad 1 & $90 \%$ & $10 \%$ \\
\hline $\operatorname{Ad} 2$ & $60 \%$ & $40 \%$ \\
\hline $\operatorname{Ad} 3$ & $70 \%$ & $30 \%$ \\
\hline $\operatorname{Ad} 4$ & $80 \%$ & $20 \%$ \\
\hline $\operatorname{Ad} 5$ & $50 \%$ & $50 \%$ \\
\hline $\operatorname{Ad} 6$ & $90 \%$ & $10 \%$ \\
\hline $\operatorname{Ad} 7$ & 0 & $100 \%$ \\
\hline $\operatorname{Ad} 8$ & $50 \%$ & $50 \%$ \\
\hline $\operatorname{Ad} 9$ & $50 \%$ & $50 \%$ \\
\hline $\operatorname{Ad} 10$ & $40 \%$ & $60 \%$ \\
\hline
\end{tabular}

Viewing Time. To evaluate whether the viewing time can be used as an implicit feedback, we compute the Spearman's Rank Correlation Coefficient between audiences' viewing time and subjective rating. The result is 0.7 , showing a strong correlation between audiences' viewing time and subjective rating. Then we can conclude that audiences' viewing time can be used as an implicit feedback to infer audiences' preference on public display advertisements.

Advertisement Recommendation Strategies. We compared our strategy for group rating (NAR) with another one, which we called it Neighbor Pearson Attractive Rating (NPAR) here. In NPAR, instead of media similarity metric, we use the value of pearson correlation coefficient between $i_{t}$ and $i_{n}$ to calculate the group rating for $i_{t}$ in step 3 . Thus we get four group advertising strategies, VT-NAR, AP-NAR, VT-NPAR, AP-NPAR.

We evaluated the two recommendation strategies separately. Groups are chosen randomly. The numbers of group member are 2, 3, 4, 5, and 6, respectively. There are 861 groups for each group size. For each group size, we calculate the average RMSE of multiple experiments as:

$$
\text { rmse }_{n}=\frac{1}{\left|G_{n}\right|} \sum_{g \in G_{n}} \sqrt{\frac{\sum_{i \in I}\left(r_{g, i}-\bar{r}_{g, i}\right)^{2}}{|I|}}
$$

where $r m s e_{n}$ denotes the RMSE value when the number of group member is $n, G_{n}$ denotes the set of group with $n$ member, and $\left|G_{n}\right|$ denotes the number of group with $\mathrm{n}$ members, $r_{g, i}$ denotes the rating for advertisement $i$ of group $g$ which we calculated, and $\bar{r}_{g, i}$ denotes the average subjective rating for advertisement $i$ of group $g$. $I$ denotes the set of advertisement candidates, and $|I|$ denote the number of advertisement in candidate set. 
Furthermore, we compute normalize Discounted Cumulative Gain (nDCG) value to evaluate the rank of advertisement which we predicted. In our experiment, we use group members' average subjective rating as the graded relevance. More detail about nDCG, please refer to [15].

As the same with RMSE, we compute the average $\mathrm{nDCG}$ value of the groups, which is given by

$$
n D C G=\frac{\sum_{g \in G_{n}} n D C G_{g, p}}{\left|G_{n}\right|}
$$

where ${ }_{n} D C G$ denotes the average $\mathrm{nDCG}$ value for 861 groups we have chosen. $g$ denotes a group. $p$ denotes the position and here our $p$ is 10 .

The nDCG result of group advertising strategies can be seen in Fig. 2. Also, from Fig. 2, we can find that all of the strategies have high $\mathrm{nDCG}$ values at about 0.8 , and the differences of nDCG values for four advertising strategies are very weak, which demonstrates that we have an advertisements ranking with high quality using all of the strategies. That is to say, our advertisement strategies can indeed infer audiences' group interest in display advertisement effectively. On the other hand, the RMSE result of group advertising strategies can be seen in Fig. 3. From Fig. 3, we can conclude that:

- The RMSE values remain stable and small with the increase of the number of group member, which shows that all of the strategies are effective for display advertisement.

- The RMSE values of the proposed strategies, i.e. AP-NAR and VT-NAR, are both smaller than AP-NPAR and VT-NPAR, which shows that media similarity degree which we proposed has a better performance than pearson correlation coefficient in public display advertisement.

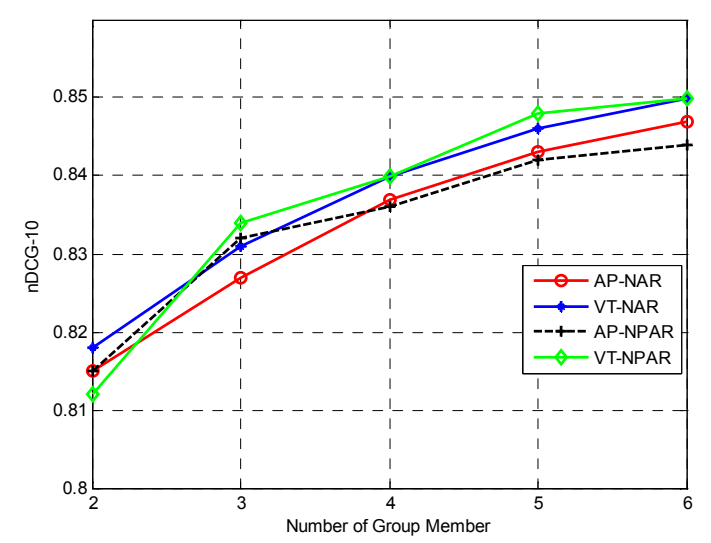

Fig. 2. Average $\mathrm{nDCG}$ versus different number of group member. Four advertising strategies are showed.

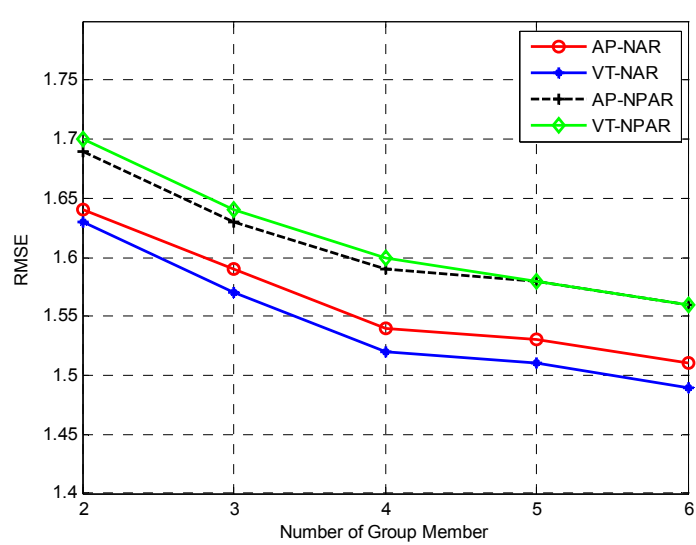

Fig. 3. Average RMSE versus different number of group member. Four advertising strategies are showed.

\section{Conclusion}

In this paper we address the public display advertisement in malls, office buildings, elevators or some other public areas. We propose to use users' viewing time to infer audiences' interest in the advertisement. In the proposed scheme, we use two metrics to infer audiences' interest, in particular, we propose a novel UCB-like learning method to infer a user's interest. The method has very low computation complexity and fits display advertisement perfectly. Furthermore, we design practical algorithms to handle the case that multiple users arrive and watch the public display at the same time. To derive a group's rating from each member's interest, we consider the media display structure characteristics of advertisements, since users' viewing of public display is highly related to the advertisement's media display structure. Experimental results show that it is accurate to infer a user's interest in a public display advertisement from her viewing time, the proposed group advertisement strategies can obtain good user advertising experience. 


\section{Acknowledgments}

The authors would like to thank all the volunteers for the experiment. And we are grateful acknowledge the support of the Fundamental Research Funds for the Central Universities.

\section{References}

[1] P. Auer, N. Cesa-Bianchi, Y. Freund, \& R. E. Schapire, The nonstochastic multiarmed bandit problem. SIAM Journal on Computing, vol. 32, no. 1, pp. 48-77, 2002.

[2] G. Bradski, A. Kaehler, Learning OpenCV: Computer vision with the OpenCV library. " O'Reilly Media, Inc.", 2008.

[3] Broder, M. Ciaramita, M. Fontoura, E. Gabrilovich, V. Josifovski, D. Metzler, \& V. Plachouras, To swing or not to swing: learning when (not) to advertise. In Proc. of the 17th ACM conference on Information and knowledge management, pp. 1003-1012. ACM. 2008.

[4] K. S. P. Chang, C. M. Danis, \& R. G. Farrell, Lunch line: using public displays and mobile devices to encourage healthy eating in an organization. In Proc. of the International Joint Conference on Pervasive and Ubiquitous Computing, pp. 823-834. ACM, 2014.

[5] Y. L. Chen, L. C. Cheng, \& C. N. Chuang, A group recommendation system with consideration of interactions among group members. Expert systems with applications, pp. 2082-2090, 2008.

[6] H. Cheng, R. V. Zwol, J. Azimi, E. Manavoglu, R. Zhang, Y. Zhou, \& V. Navalpakkam, Multimedia features for click prediction of new ads in display advertising. In Proc. of the 18th SIGKDD, pp. 777-785. ACM, 2012.

[7] P. Dias, J. Magalhães, Music recommendations for groups of users. In Proc. of the 2013 ACM international workshop on Immersive media experiences, pp. 21-24. ACM, 2013.

[8] J. Gorla, N. Lathia, S. Robertson, \& J. Wang, Probabilistic group recommendation via information matching. pp. 495-504., WWW, 2013.

[9] G. Parra, J. Klerkx, E. Duval, Understanding engagement with interactive public displays: an awareness campaign in the wild. In Proc. Of the International Symposium on Pervasive Displays, ACM, 2014.

[10] J. Müller, D. Eberle, K. Tollmar, Communiplay: a field study of a public display mediaspace. In Proc. of the 32nd annual ACM conference on Human factors in computing systems, pp. 1415-1424. ACM, 2014.

[11] J. She, J. Crowcroft, H. Fu, F. Li, Convergence of interactive displays with smart mobile devices for effective advertising: a survey. ACM TOMCCAP, 2014.

[12] Information on https://videopath.com/five-second-rule/

[13] L. Xiang, Recommendation system practice, POSTS and TELECOM PRESS, 2012.

[14] X. Yi, L. Hong, E. Zhong, N. N. Liu, S. Rajan, Beyond clicks: dwell time for personalization. In Proc. of the 8th ACM Conference on Recsys, pp. 113-120. ACM, 2014.

[15] Information on https://en.wikipedia.org/wiki/Discounted_cumulative_gain 